\title{
Phonon entropy of alloying and ordering of $\mathrm{Cu}-\mathrm{Au}$
}

\author{
P. D. Bogdanoff, T. L. Swan-Wood, and B. Fultz \\ California Institute of Technology, W. M. Keck Laboratory, mail 138-78, Pasadena, California 91125
}

(Received 20 January 2003; published 11 July 2003)

\begin{abstract}
Inelastic neutron scattering spectra were measured with a time-of-flight spectrometer on six disordered $\mathrm{Cu}-\mathrm{Au}$ alloys at $300 \mathrm{~K}$. The neutron-weighted phonon density of states was obtained from a conventional analysis of these spectra. Several methods were developed to account for this neutron weighting and obtain the phonon entropy of the disordered alloys. The phonon entropies of formation of disordered fcc $\mathrm{Cu}-\mathrm{Au}$ alloys obtained in this way were generally mutually consistent, and were also consistent with predictions from a cluster approximation obtained from ab-initio calculations by Ozolinšs, Wolverton, and Zunger. We estimate a phonon entropy of disordering of $0.15 \pm 0.05 k_{\mathrm{B}} /$ atom in $\mathrm{Cu}_{3} \mathrm{Au}$ at $300 \mathrm{~K}$. A resonance mode associated with the motions of the heavy $\mathrm{Au}$ atoms in the $\mathrm{Cu}$-rich alloys was observed at $9 \mathrm{meV}$. An analysis of the resonance mode provided a check on the partial phonon entropy of Au atoms.
\end{abstract}

DOI: 10.1103/PhysRevB.68.014301

PACS number(s): 78.70.Nx, 81.30.Bx, 63.20.-e

\section{INTRODUCTION}

It is universally appreciated that most of the heat in solids goes into the vibrations of atoms, and the associated phonon entropy is large. For many years, however, it was unclear if different alloy phases had sufficient differences in phonon entropy to affect their relative thermodynamic stabilities. One of the earlier controversies involved $\mathrm{Cu}_{3} \mathrm{Au}$, where the temperature dependence of the electrical resistivity was used to argue that phonon entropy strongly affected the entropy of the chemical order-disorder transition, ${ }^{1}$ whereas lowtemperature measurements of elastic constants were used to argue that the difference in phonon entropy was negligible. ${ }^{2}$ Neither measurement provided direct information on phonon entropy, however. Over the past decade the experimental and theoretical efforts have become more focused, and a number of cases were found where phonon entropy is significant in structural and chemical phase transitions in alloys. ${ }^{3-13}$ The origins of these differences in phonon entropy are now an active topic of research.

The $\mathrm{Cu}-\mathrm{Au}$ system has long been a testbed for studies of alloy phases and order-disorder phase transitions. At three stoichiometric compositions, the stable phases at low temperature are ordered intermetallic compounds having the $L 1_{2}$ structures for $\mathrm{Cu}_{3} \mathrm{Au}$ and $\mathrm{CuAu}_{3}$, and $L 1_{0}$ for $\mathrm{CuAu}$. At elevated temperatures, above a critical temperature of $663 \mathrm{~K}$ $\left(390{ }^{\circ} \mathrm{C}\right.$ ) for $L 1_{2}$ for $\mathrm{Cu}_{3} \mathrm{Au}$ for example, $\mathrm{Cu}$ and $\mathrm{Au}$ form a face-centered-cubic (fcc) single-phase solid solution at all compositions. The chemical order of the intermetallic compounds can be suppressed by rapidly quenching the alloys from high temperature. The effects of ordering on numerous properties have been reported, including elastic constants ${ }^{2}$, heat capacity ${ }^{14}$ and lattice dynamics ${ }^{15}$. A property of an ordered phase is typically compared to that of a disordered phase with the same composition. When doing so, it is often found that the properties of the disordered alloy are the more difficult to understand. Without the simplifications that follow from Bloch's theorem, and with local relaxations of atom positions, true $a b$-initio calculations are expensive and methodologically uncertain, but progress has been made. ${ }^{13,16-18}$ The energy difference between the ordered and disordered phases can be estimated by modern electronic structure calculations, and the configurational entropy is well understood through cluster-variation calculations or Monte Carlo simulations. The phonon component of the transition entropy, $\Delta S_{\mathrm{ph}}^{\mathrm{d}-\mathrm{o}}=S_{\mathrm{ph}}^{\mathrm{dis}}-S_{\mathrm{ph}}^{\text {ord }}$, where $S_{\mathrm{ph}}^{\mathrm{dis}}$ and $S_{\mathrm{ph}}^{\text {ord }}$ are the phonon entropies of the disordered and ordered phases, is a challenge for theoretical calculations. Ozoliņšs, Wolverton, and Zunger used an inverse cluster-variation method with $a b$ inito calculations of the lattice dynamics of ordered $\mathrm{Cu}-\mathrm{Au}$ phases to obtain a small $\Delta S_{\mathrm{ph}}^{\mathrm{d}-\mathrm{o}}$ value of $0.06 k_{\mathrm{B}} /$ atom at $300 \mathrm{~K}^{8}{ }^{8}$ In experimental work using low-temperature calorimetry, Nagel, Anthony, and Fultz obtained a value of $\Delta S_{\mathrm{ph}}^{\mathrm{d}-\mathrm{o}}$ of $0.14 \pm 0.05 k_{\mathrm{B}}$ /atom for $\mathrm{Cu}_{3} \mathrm{Au}$ at $300 \mathrm{~K}$. ${ }^{19}$ They also used a Born-von Kármán model with force constants from phonon dispersion measurements of the ordered and disordered phases ${ }^{15}$ to obtain a $\Delta S_{\mathrm{ph}}^{\mathrm{d}-\mathrm{o}}$ of $0.23 k_{\mathrm{B}} /$ atom at $300 \mathrm{~K}$. This calculation of the phonon density of states (DOS) suffers seriously from the use of the virtual crystal approximation to interpret the disordered phase lattice dynamics, however.

In the present investigation, we used a time-of-flight inelastic neutron spectrometer to measure the differences in phonon scattering from disordered $\mathrm{Cu}_{3} \mathrm{Au}$, obtaining data matched for comparison with prior work on ordered $\mathrm{Cu}_{3} \mathrm{Au} .{ }^{20}$ Unfortunately, neutrons are scattered up to 3.21 times more strongly by phonons whose dominant motions involve $\mathrm{Cu}$ atoms, compared to phonons involving motions of $\mathrm{Au}$ atoms. This difference is responsible for a "neutronweight" distortion of the phonon DOS. Until recently, there has been little work to overcome this basic characteristic of phonon scattering. When the Born-von Kármán model can be utilized to simulate the lattice dynamics, as is the case for the ordered phases, it is possible to calculate the neutron weight correction as was done for $L 1_{2}$-ordered $\mathrm{Cu}_{3} \mathrm{Au}$ (Ref. 20) and $B 2$-ordered NiTi. ${ }^{10}$ Sometimes it is possible to iterate the interatomic force constants to optimize the match between calculated and experimental inelastic scattering, and use the force constants so obtained for a neutron weight correction, as was done for $L 1_{2}$-ordered $\mathrm{Pt}_{3} \mathrm{Fe}^{21}$ Chemical disorder in solid solutions of $\mathrm{Cu}-\mathrm{Au}$ removes translational peri- 
odicity, precluding neutron-weight corrections with a Bornvon Kármán model. The central focus of the present research is developing a reliable methodology for obtaining the phonon entropy from the neutron-weighted phonon DOS, and not the phonon DOS itself. Several neutron-weight corrections are developed and tested, including a comparison with effective cluster interactions from an ab initio theoretical study. We report phonon entropies of formation of disordered $\mathrm{Cu}-\mathrm{Au}$ alloys, and the consistency of these results with each other and with theoretical results ${ }^{8}$ indicates their reliability. We also report a difference in phonon entropy of disordered and ordered $\mathrm{Cu}_{3} \mathrm{Au}$ of $0.15 \pm 0.05 k_{\mathrm{B}} /$ atom at $300 \mathrm{~K}$.

\section{EXPERIMENTAL METHODS AND RESULTS}

Pieces of $\mathrm{Au}$ and $\mathrm{Cu}$, both of $99.99 \%$ purity, were arcmelted to provide ingots with composition $\mathrm{Cu}_{x} \mathrm{Au}_{1-x}$, where the values of $x$ were $96,82,75,68,50$, and 25 at $\%$. The total mass lost during arc-melting was negligible, and there was no evidence of a surface oxide. Each ingot was cold-rolled to a thickness of approximately $0.76 \mathrm{~mm}$. The cold-rolled strips were cut into smaller pieces, annealed for $45 \mathrm{~min}$ at $1123 \mathrm{~K}$ $\left(850^{\circ}\right)$ in evacuated quartz ampoules, then quenched into iced brine to preserve chemical disorder. X-ray diffractometry with Co $K \alpha$ radiation and an Inel CPS-120 position sensitive detector showed no evidence of chemical longrange order in the quenched specimens. ${ }^{20}$ The presence of short-range order (SRO) is harder to rule out, but the quenched samples showed no modulations in the background around the positions of the superlattice diffractions. A slow quench from above the critical temperature can produce samples with partial SRO however, ${ }^{22}$ typically when the quenched samples have equiaxed shapes of approximately $1 \mathrm{~cm}$ dimension. ${ }^{23}$ Cooling rates of hundreds of degrees $\mathrm{K}$ per second largely suppress changes in SRO in $\mathrm{Cu}_{3} \mathrm{Au} .{ }^{24}$ Such rates may be achieved by our quenching method, which did succeed in producing significant differences between the ordered and disordered samples of $\mathrm{Cu}_{3} \mathrm{Au}$, as reported below.

Inelastic neutron scattering spectra were measured with the time-of-flight (TOF) chopper spectrometer LRMECS, at the IPNS spallation neutron source at Argonne National Laboratory. All samples were assembled as a mosaic plate of $7 \times 9 \mathrm{~cm}$, and encased in thin-walled $\mathrm{Al}$ pans for mounting in the displex refrigerator of the LRMECS spectrometer. The plates and powder samples were mounted at a $45^{\circ}$ angle normal to the incident beam to minimize self shielding. Spectra with an incident energy $E_{\mathrm{i}}$ of $35 \mathrm{meV}$ were measured at $300 \mathrm{~K}$ for all samples and an empty container. A run with a "black absorber" cadmium sheet in the sample position was measured at room temperature to improve the background correction. Detector response was calibrated with a run performed on a vanadium sample at room temperature, using broadband radiation without a Fermi chopper. The continous detector coverage on LRMECS, ranging from $3^{\circ}$ to $117^{\circ}$, allowed a simultaneous probe of the spectral response over a large range of momentum and energy transfer

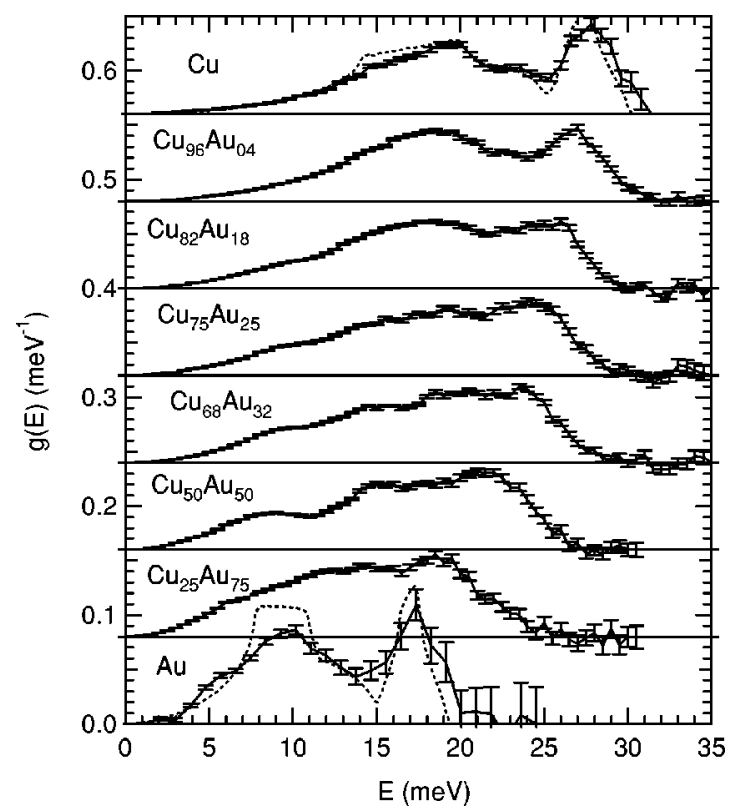

FIG. 1. Neutron-weighted phonon DOS of disordered $\mathrm{Cu}_{1-x} \mathrm{Au}_{x}$ alloys. Thin dashed curves for elemental $\mathrm{Cu}$ and $\mathrm{Au}$ were calculated using force constants of Ref. 34 and 18.

$(\vec{Q}, \hbar \omega)$, with $|\vec{Q}(\omega)|$ varying between 0.2 and $7 \AA^{-1}$ at zero energy transfer.

All TOF spectra were first normalized and corrected for detector efficiency and time-independent background following standard procedures. In this step, the detectors were combined into 12 groups spanning $10^{\circ}$ each with average angles from $5^{\circ}$ to $115^{\circ}$. Scattering from the container and spectrometer hardware was determined using the runs on the empty container and $\mathrm{Cd}$ sample, taking into account the sample absorption. The data below 3-4 $\mathrm{meV}$ are dominated by the large elastic peak, which was stripped from the data. The inelastic scattering at these low energies is featureless and linear in the hydrodynamic limit, and is estimated easily. The neutron-weighted phonon density of states was determined from the corrected spectra with the procedure described previously. ${ }^{20}$ This procedure is an iterative one, where a DOS curve is assumed, from which the single phonon and multiphonon scatterings are then calculated. Subtracting the multiphonon scattering from the background-corrected data leaves the single-phonon scattering, from which a new phonon DOS is obtained by a thermal correction. Typically the data require only two iterations before self-consistency is found for the DOS curve (or for alloys the neutron-weighted DOS curve).

The final neutron-weighted phonon DOS curves of $\mathrm{Cu}_{1-x} \mathrm{Au}_{x}$ are shown in Fig. 1. These curves change continuously with decreasing $\mathrm{Cu}$ concentration. Two changes are most evident. The DOS softens monotonically with increasing Au concentration. This change can be seen most clearly in the high energy cutoff of the DOS, which is $31 \mathrm{meV}$ for $\mathrm{Cu}$ and $20 \mathrm{meV}$ for $\mathrm{Au}$. The effect is not quite linear in $\mathrm{Au}$ concentration, as can be observed in Fig. 1-the DOS of $\mathrm{Cu}_{50} \mathrm{Au}_{50}$ terminates at $27 \mathrm{meV}$, not at $25.5 \mathrm{meV}$. Second, a peak at $9 \mathrm{meV}$ grows rapidly with increasing Au concentra- 
tion. This peak is almost certainly a resonance mode due to Au vibrations.

\section{DISCUSSION}

\section{A. Neutron weighting}

The experimental curves of Fig. 1 could be distorted by neutron weighting from the shapes of the true phonon DOS curves, perhaps significantly. The ratios $\sigma_{\mathrm{Cu}} / m_{\mathrm{Cu}}$ and $\sigma_{\mathrm{Au}} / m_{\mathrm{Au}}$, which are the most important factors controlling the relative efficiencies of phonon scattering by $\mathrm{Cu}$ and $\mathrm{Au}$ atoms, differ by a factor of 3.21. This implies that some parts of the neutron-weighted DOS, if associated with motions of $\mathrm{Au}$ atoms, could be underweighted by a factor as large as 3.21 compared to the true phonon DOS. Furthermore, the neutron-weight correction is different for each of the six alloys. (There is, of course, no neutron weight correction for the pure elements $\mathrm{Cu}$ and $\mathrm{Au}$.)

In the quasi-harmonic approximation, the phonon DOS at temperature $T$ and volume $V, g_{T, V}(E)$, provides the phonon entropy, $S_{\mathrm{ph}}(T)$,

$$
\begin{aligned}
S_{\mathrm{ph}}(T)= & -3 k_{\mathrm{B}} \int_{0}^{\infty} g_{T, V}(E)\left[\left(n_{E}+1\right) \ln \left(n_{E}+1\right)\right. \\
& \left.-n_{E} \ln \left(n_{E}\right)\right] \mathrm{d} E,
\end{aligned}
$$

where $n_{E}$ is the phonon occupancy factor. Unfortunately, we do not measure $g_{T, V}(E)$ directly, but spectra as in Fig. 1 are neutron-weighted DOS curves, $g_{T, V}^{\mathrm{nw}}(E)$. This $g_{T, V}^{\mathrm{nw}}(E)$ is composed of neutron-weighted contibutions from the partial DOS curves of elements, $g^{d}(E)$, where " $d$ " denotes $\mathrm{Cu}$ or $\mathrm{Au}$,

$$
g_{T, V}^{\mathrm{nw}}(E) \propto \exp (2 W) \sum_{d} g^{d}(E) \exp \left(-2 W_{d}\right) \frac{\sigma_{d}}{m_{d}},
$$

and $\exp \left(-2 W_{d}\right)$ is the Debye-Waller factor of atom $d$. The factor $\exp (2 W)$ is the average Debye-Waller correction calculated from the self-consistent neutron-weighted DOS. In what follows we assume $\exp \left[-2\left(W-W_{d}\right)\right]=1$, an assumption that is most reliable at low temperatures. In this case we can write simply

$$
g_{T, V}^{\mathrm{nw}}(E)=C \sum_{d} g^{d}(E) \frac{\sigma_{d}}{m_{d}} x_{d},
$$

where the normalization, $C$, depends on concentrations of the elements and their scattering strengths,

$$
C=\left(\sum_{d} \frac{\sigma_{d}}{m_{d}} x_{d}\right)^{-1}
$$

If we evaluate Eq. 1 using the neutron-weighted DOS, $g_{T, V}^{\mathrm{nw}}(E)$, instead of the true phonon DOS, $g_{T, V}(E)$, we obtain a "neutron-weighted phonon entropy," $S_{\mathrm{ph}}^{\text {nw }}(T)$, where

$$
\begin{aligned}
S_{\mathrm{ph}}^{\mathrm{nw}}(T) \equiv & -3 k_{\mathrm{B}} \int_{0}^{\infty} g_{T, V}^{\mathrm{nw}}(E)\left[\left(n_{E}+1\right) \ln \left(n_{E}+1\right)\right. \\
& \left.-n_{E} \ln \left(n_{E}\right)\right] \mathrm{d} E .
\end{aligned}
$$

This $S_{\mathrm{ph}}^{\mathrm{nw}}(T)$ proves useful because of how it is related to the different $g^{d}(E)$. Substituting Eq. (3) into Eq. (5)

$$
\begin{gathered}
S_{\mathrm{ph}}^{\mathrm{nw}}=-3 k_{\mathrm{B}} C \sum_{d} \frac{\sigma_{d}}{m_{d}} x_{d} \int_{0}^{\infty} g^{d}(E)\left[\left(n_{E}+1\right) \ln \left(n_{E}+1\right)\right. \\
\left.-n_{E} \ln \left(n_{E}\right)\right] \mathrm{d} E \\
S_{\mathrm{ph}}^{\mathrm{nw}}=C \sum_{d} \frac{\sigma_{d}}{m_{d}} x_{d} S_{\mathrm{ph}}^{d}(T)
\end{gathered}
$$

where $S_{\mathrm{ph}}^{d}(T)$ is the phonon entropy of atom $d$.

The neutron-weighted entropy is thus a sum of the partial entropies, $S_{\mathrm{ph}}^{d}(T)$, weighted by the phonon scattering strengths of the atoms, $d$. Equation (7) is analogous to the phonon entropy, which equals the sum of the phonon partial entropies of all species,

$$
S_{\mathrm{ph}}(T)=\sum_{d} S_{\mathrm{ph}}^{d}(T),
$$

where each partial phonon entropy, $S_{\mathrm{ph}}^{d}(T)$, is related to a normalized phonon partial DOS, $g^{d}(E)$, in the usual way

$$
\begin{aligned}
S_{\mathrm{ph}}^{d}(T)= & -3 k_{\mathrm{B}} \int_{0}^{\infty} g^{d}(E)\left[\left(n_{E}+1\right) \ln \left(n_{E}+1\right)\right. \\
& \left.-n_{E} \ln \left(n_{E}\right)\right] \mathrm{d} E .
\end{aligned}
$$

For the case of the binary alloy $\mathrm{Cu}_{1-x} \mathrm{Au}_{x}$, Eqs. (8) and (7) are

$$
\begin{gathered}
S_{\mathrm{ph}}(x)=(1-x) S_{\mathrm{ph}}^{\mathrm{Cu}}(x)+x S_{\mathrm{ph}}^{\mathrm{Au}}(x), \\
S_{\mathrm{ph}}^{\mathrm{nw}}(x)=C^{\prime}\left(3.21(1-x) S_{\mathrm{ph}}^{\mathrm{Cu}}(x)+x S_{\mathrm{ph}}^{\mathrm{Au}}(x)\right),
\end{gathered}
$$

where $x$ is Au concentration, and $0 \leqslant x \leqslant 1$. Here the normalization constant $C^{\prime}$ is

$$
C^{\prime}=[3.21(1-x)+x]^{-1} .
$$

In the present work we do not attempt to correct the $g_{T, V}^{\mathrm{nw}}(E)$ because obtaining a phonon DOS would require an analysis of the lattice dynamics of the disordered alloy. Instead, we infer the true partial entropies $S_{\mathrm{ph}}^{\mathrm{Cu}}(x)$ and $S_{\mathrm{ph}}^{\mathrm{Au}}(x)$ of Eq. (10) from measured neutron-weighted entropies $S_{\mathrm{ph}}^{\mathrm{nw}}(x)$, and from these we obtain corrected phonon entropies, $S_{\mathrm{ph}}(x)$.

\section{B. Neutron deweighting in the point approximation and its modification}

We seek true phonon entropies from neutron-weighted phonon entropies. The simplest chemical model of phonon entropy assumes 


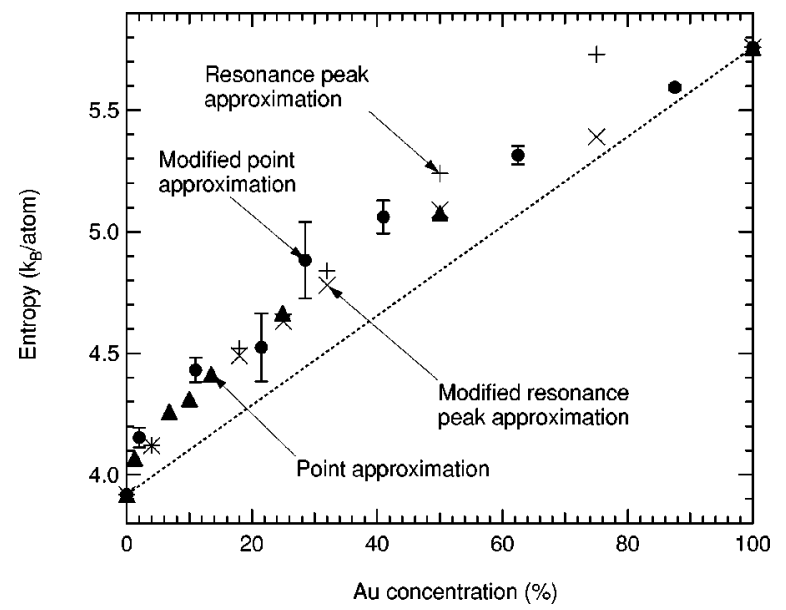

FIG. 2. Phonon entropies of disordered $\mathrm{Cu}-\mathrm{Au}$ alloys at $300 \mathrm{~K}$, corrected for neutron weighting by the methods indicated in labels. The straight dashed line is the straight line connecting the phonon entropies of elemental $\mathrm{Au}$ and $\mathrm{Cu}$.

$$
S_{\mathrm{ph}}(x)=(1-x) S_{\mathrm{ph}}^{\mathrm{Cu}}+x S_{\mathrm{ph}}^{\mathrm{Au}},
$$

where neither $S_{\mathrm{ph}}^{\mathrm{Cu}}$ nor $S_{\mathrm{ph}}^{\mathrm{Au}}$ are functions of Au concentration, $x$. Equation (13) has a form similar to that of 10, and these two equations would be identical if $S_{\mathrm{ph}}^{\mathrm{Cu}}$ and $S_{\mathrm{ph}}^{\mathrm{Au}}$ were not functions of $x$. In this case the neutron-weighted phonon DOS at a particular composition would be identical to the true phonon DOS at a different composition (having more $\mathrm{Cu}$ ). Compatibility of Eqs. (10) and (13) is then possible by defining a composition $x^{\text {nw }}$ that weights more heavily the $\mathrm{Cu}$ atoms:

$$
x^{\mathrm{nw}}=\frac{x}{x+3.21(1-x)},
$$

so that

$$
S_{\mathrm{ph}}^{\mathrm{nw}}(x)=\left(1-x^{\mathrm{nw}}\right) S_{\mathrm{ph}}^{\mathrm{Cu}}+x^{\mathrm{nw}} S_{\mathrm{ph}}^{\mathrm{Au}} .
$$

Comparing Eqs. (15) and (13),

$$
S_{\mathrm{ph}}\left(x^{\mathrm{nw}}\right)=S_{\mathrm{ph}}^{\mathrm{nw}}(x) .
$$

The phonon entropy, corrected in this "point approximation," is shown in Fig. 2. The point approximation is strictly correct only if the partial entropies (and partial DOS) of $\mathrm{Au}$ and $\mathrm{Cu}$ are unchanged as the alloy varies in composition between $x$ and $x^{\mathrm{nw}}$. When there are large differences between $x$ and $x^{\mathrm{nw}}$, this approximation is likely to have large errors.

A "modified point approximation" improves upon the previous point approximation by measuring $S_{\mathrm{ph}}^{\mathrm{nw}}$ at two similar compositions, then solving two equations of the form of Eq. (15). The partial entropies, $S_{\mathrm{ph}}^{\mathrm{Cu}}$ and $S_{\mathrm{ph}}^{\mathrm{Au}}$ calculated this way are an average of similar partial entropies at the two alloy compositions. For example, using the neutron-weighted entropies of the $\mathrm{Cu}_{68} \mathrm{Au}_{32}$ and $\mathrm{Cu}_{50} \mathrm{Au}_{50}$ alloys, we calculate the average partial entropies $S_{\mathrm{ph}}^{\mathrm{Cu}}=4.12 k_{\mathrm{B}}$ and $S_{\mathrm{ph}}^{\mathrm{Au}}$ $=6.41 k_{\mathrm{B}}$. From these values we calculate the phonon entropy of the alloy at composition $\mathrm{Cu}_{59} \mathrm{Au}_{41}$. The partial entropies of $\mathrm{Au}$ and $\mathrm{Cu}$, calculated from pairs of adjacent com-

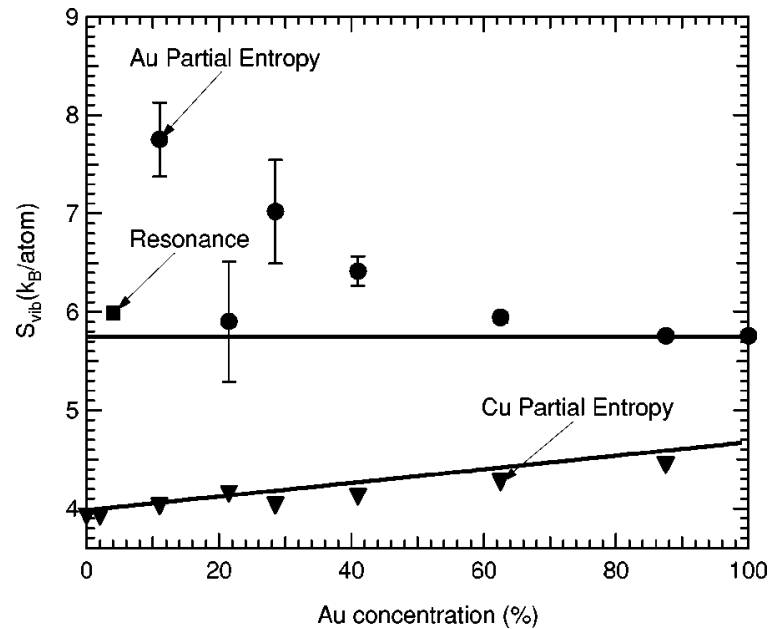

FIG. 3. Partial phonon entropies of $\mathrm{Cu}$ and $\mathrm{Au}$ atoms in $\mathrm{Cu}-\mathrm{Au}$ alloys. Points were obtained in the modified point approximation, except point labeled "Resonance," which was from the Au resonance peak approximation. [Lines were obtained with a threeparameter generalized partial entropy model. (Ref. 35)]

positions, are shown in Fig. 3. The closer are the two alloys in composition, the more accurate the assumption of equal partial entropies. Unfortunately, as the compositions become closer in value, it becomes more difficult to resolve accurately the difference in neutron-weighed entropies, given the statistical errors in the experimental spectra. The large error bars on the total entropy values at $\mathrm{Cu}$ concentrations of $78.5 \%$ and $71.5 \%$, shown in Fig. 2, result from the small differences between the neutron-weighted entropies at alloy compositions $\mathrm{Cu}_{82} \mathrm{Au}_{18}, \mathrm{Cu}_{75} \mathrm{Au}_{25}$, and $\mathrm{Cu}_{68} \mathrm{Au}_{32}$. This is not a fundamental flaw with the approach, but it does require that the experimental data be of high quality.

\section{Neutron deweighting with the local cluster approximation}

The local cluster approximation (LCA) is used widely for calculating ground-state thermodynamic properties of chemically disordered materials. ${ }^{17,25}$ It can be used to parametrize the partial entropies of $\mathrm{Cu}$ and $\mathrm{Au}$ in disordered $\mathrm{Cu}-\mathrm{Au}$ alloys. In the LCA the phonon entropy $S_{\text {vib }}$ is

$$
S_{\mathrm{ph}}=S_{0}+S_{1} \sum_{\sigma_{1}} \phi\left(\sigma_{1}\right)+S_{2} \sum_{\sigma_{2}} \phi\left(\sigma_{2}\right)+S_{3} \sum_{\sigma_{3}} \phi\left(\sigma_{3}\right)+\ldots,
$$

where the $S_{i}$ are the cluster coefficients associated with cluster $i, \sigma_{i}$ is the $i^{\text {th }}$ cluster type and $\phi\left(\sigma_{i}\right)$ is the spin-product over cluster $\sigma_{i}$. Cluster expansions have proved useful for analysis of previous experimental results. ${ }^{20,26}$ For $g_{\mathrm{ph}}^{\mathrm{nw}}(E)$ measured on fully disordered alloys, however, this parameterization cannot be used for reliable inversions to extract partial entropies. [For fully disordered alloys, the LCA basis functions become linearly-dependent beyond the point cluster. For example, probabilities for a first-nearest neighbor ( $1 \mathrm{nn})$ pair are the same as for a $2 \mathrm{nn}$ pair.] On the other hand, 
TABLE I. LCA cluster coefficients at $300 \mathrm{~K}$ from Ozoliņs $\breve{s}$, Wolverton, and Zunger. Units are in $k_{\mathrm{B}}$ /atom. The cluster expansion is for the phonon entropy of formation of disordered $\mathrm{Cu}-\mathrm{Au}$ alloys. (Refs. 8 and 27)

\begin{tabular}{cccccc}
\hline \hline & $S_{0}$ & $S_{1}$ & $S_{2}$ & $S_{3}$ & $S_{4}$ \\
\hline Cluster coefficients & 0.234 & 0.0185 & -0.108 & -0.122 & -0.0185 \\
\hline \hline
\end{tabular}

if the effective cluster interactions for phonon entropy are available, they can be tested against the experimental neutron-weighted DOS.

Ozolinşs, Wolverton, and Zunger ${ }^{8}$ recently performed $a b$ initio electronic-structure calculations of the phonon DOS and phonon entropy for a series of ordered $\mathrm{Cu}-\mathrm{Au}$ alloys. Using results from six ordered $\mathrm{Cu}-\mathrm{Au}$ alloys, they obtained a set of cluster-coefficients $\left\{S_{i}\right\}$ that best reproduced their results in Eq. (17). These clusters were the empty cluster $S_{0}$, the point cluster $S_{1}$, the $1 \mathrm{nn}$ pair $S_{2}$, the $2 \mathrm{nn}$ pair $S_{3}$ and the 1nn triangle $S_{4}$. The values for the cluster coefficients at $300 \mathrm{~K}$, given in Table I, were kindly provided by Ozolins $\breve{s}^{27}$ Using the values given in Table I and Eq. (17), we can calculate the phonon entropy of formation of binary $\mathrm{Cu}_{1-x} \mathrm{Au}_{x}$ alloys of arbitrary $\mathrm{Cu}$ concentrations. For the case of a fully disordered binary system, Eq. (17) simplifies to

$$
S_{\mathrm{ph}}^{\mathrm{form}}=S_{0}+S_{1}(1-2 x)+\left(S_{2}+S_{3}\right)(1-2 x)^{2}+S_{4}(1-2 x)^{3} .
$$

Note that we include only five terms corresponding to the five clusters in our LCA sum. The terms corresponding to the $1 \mathrm{nn}$ pair and $2 \mathrm{nn}$ pairs, $S_{2}$ and $S_{3}$, have the same functional dependence on $x$ (because for fully disordered alloys the probabilities for $1 \mathrm{nn}$ and $2 \mathrm{nn}$ pairs are equal.) We evaluated Eq. (18) and show the results in Fig. 4. These LCA results are in generally good agreement with the results from our analyses of the experimental spectra.

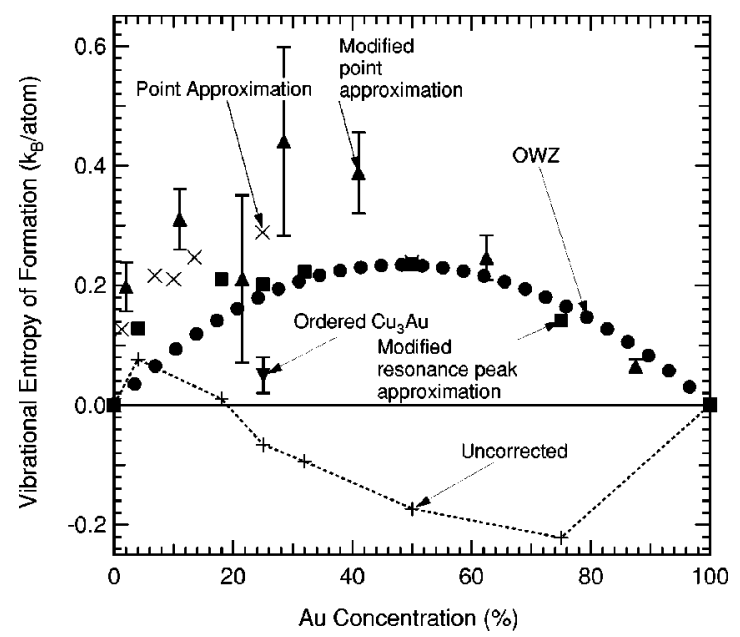

FIG. 4. Vibrational entropy of formation of disordered $\mathrm{Cu}-\mathrm{Au}$ alloys at $300 \mathrm{~K}$. Labels refer to neutron-weight corrections described in the text. The circles marked OWZ are the LCA results calculated with parameters used by Ozolinšs, Wolverton, and Zunger. (Refs. 8, and 27).

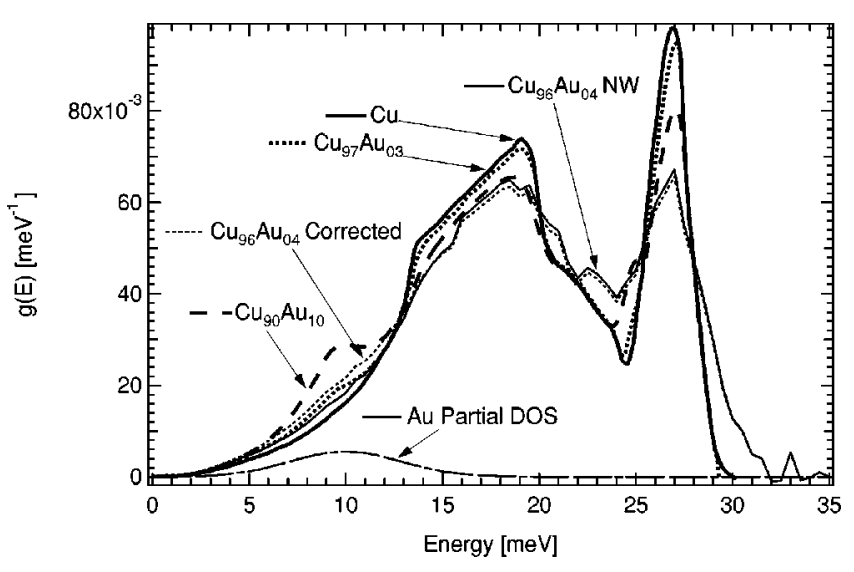

FIG. 5. Phonon DOS curves for $\mathrm{Cu}-\mathrm{Au}$ alloys of dilute Au concentration. The experimental curves include the neutron-weighted DOS, Au partial DOS, and corrected DOS of $\mathrm{Cu}_{96} \mathrm{Au}_{04}$ alloy with the resonance peak approximation. The phonon DOS of $3 \%$ and $10 \% \mathrm{Cu}-\mathrm{Au}$ alloys were calculated by Elliott and Taylor (Ref. 33). The $\mathrm{Cu}$ curve was calculated using force constants of Ref. 18 .

\section{Au resonance mode and neutron deweighting}

For $\mathrm{Au}$ concentrations between 0 and $50 \%$, the peak at $9 \mathrm{meV}$ grows with increasing Au concentration. Similar phenomena have been reported in phonon dispersion measurements on $\mathrm{Cu}-\mathrm{Au}$ (Ref. 28) and Cr-W (Refs. 29 and 30) dilute alloys. The low-energy peaks in dilute $\mathrm{Cu}-\mathrm{Au}$ alloys are consistent with the resonance mode ${ }^{16,31}$ predicted by the massdefect theory of lattice dynamics. ${ }^{32}$ A resonance mode comprises damped vibrational motions of heavy atoms, with contributions from the adjacent light atoms. With increasing concentration of the heavy atom, the partial DOS of the heavy atom broadens and develops more features. Elliott and Taylor $^{33}$ calculated the phonon DOS of $3 \%$ and $10 \% \mathrm{Cu}-\mathrm{Au}$ alloys using an analytical Green-function technique. Their results, presented in Fig. 5, show a low-energy resonance peak at $9 \mathrm{meV}$ that grows proportionally with Au concentration. This peak was approximated adequately as a Gaussian function having a standard deviation of $2.5 \mathrm{meV}$. Using this peak for the $\mathrm{Au}$ partial DOS, $g^{\mathrm{Au}}(E)$, we account for its neutron de-weighting and extract the $\mathrm{Cu}$ partial DOS, $g^{\mathrm{Cu}}(E)$ from the measured $g_{T, V}^{\mathrm{nw}}(E)$. [Although not shown in Fig. 5, the $\mathrm{Cu}$ partial DOS is nearly identical to the neutronweighted DOS, $g_{T, V}^{\text {nw }}(E)$.] We then obtained the total DOS, $g(x, E)$, at each alloy composition. Results for the alloy composition $\mathrm{Cu}_{96} \mathrm{Au}_{04}$ are shown in Fig. 5. Using these total DOS curves, we obtained the phonon entropies shown in Figs. 2 and 4. At low Au concentrations, the entropies in the present "resonance-peak approximation" are in excellent agreement with the values from the point approximation and the modified point approximation. These results were largely insensitive to the width of the Gaussian that approximates the Au partial DOS.

The resonance-peak approximation for the $\mathrm{Au}$ partial DOS is a poor one for alloys with high $\mathrm{Au}$ concentrations. This is apparent by looking at the DOS of elemental Au in Fig. 1, which bears little resemblance to a Gaussian in energy centered at $9 \mathrm{meV}$. With a "modified resonance-peak approximation" we improved upon our scheme by assuming 


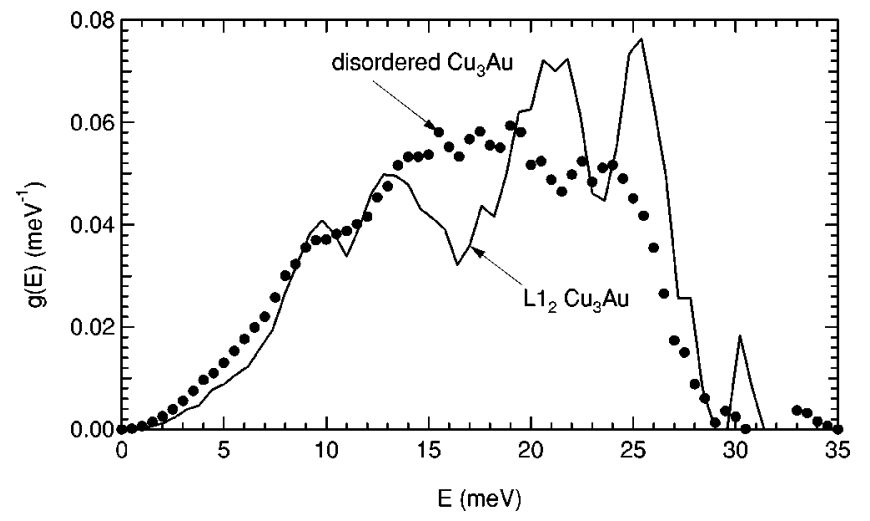

FIG. 6. Phonon DOS curves, corrected for neutron weighting of ordered and disordered $\mathrm{Cu}_{3} \mathrm{Au}$.

the Au partial DOS resembles the Gaussian resonance peak $G(E)$ at low Au concentrations, the elemental Au DOS at high $\mathrm{Au}$ concentrations, and a weighted sum of the two at intermediate $\mathrm{Au}$ concentrations,

$$
g^{\mathrm{Au}}(x, E)=(1-x) G(E)+x g_{\mathrm{ph}}^{\mathrm{Au}},
$$

where $g^{\mathrm{Au}}(x, E)$ is the partial DOS of Au within an alloy of Au concentration $x$. The phonon entropies obtained from this modified resonance-peak approximation are shown in Figs. 2 and 4.

\section{E. Vibrational entropy of ordering of $L 1_{2} \mathrm{Cu}_{3} \mathrm{Au}$}

The phonon DOS of the ordered alloy can be obtained reliably because the neutron-weight correction can be calculated using the Born-von Kármán lattice dynamics, with force constants from the measured phonon dispersions for $\mathrm{L1}_{2}$-ordered $\mathrm{Cu}_{3} \mathrm{Au}$. ${ }^{15}$ For the case of disordered $\mathrm{Cu}_{3} \mathrm{Au}$, a Born-von Kármán lattice dynamics model is not possible. For the disordered phase we used the same neutron-weight correction as for the ordered phase. Corrected phonon DOS curves of both ordered and disordered $\mathrm{Cu}_{3} \mathrm{Au}$ are shown in Fig. 6. The DOS of the ordered alloy exhibits distinct van Hove singularities at 9, 13, 20, and $25 \mathrm{meV}$. The DOS of the disordered alloy has one very broad central feature around 16 $\mathrm{meV}$, and two small peaks at 10 and $24 \mathrm{meV}$. The highenergy cutoff of the DOS is the same for the ordered and disordered alloys. Using these phonon DOS curves and Eq. (1), the phonon entropy of the disordered phase is larger than that of the ordered phase by $\Delta S_{\mathrm{ph}}^{\mathrm{d}-\mathrm{o}}=0.12 k_{\mathrm{B}} /$ atom. This result is much smaller than that obtained with the virtual crystal approximation for the disordered alloy, which gave a value of $\Delta S_{\mathrm{ph}}^{\mathrm{d}-\mathrm{o}}=0.23 k_{\mathrm{B}} /$ atom. ${ }^{19}$

Nagel et al. ${ }^{19}$ used the force constants of Katano et al. ${ }^{15}$ to evaluate the eigenvectors of the dynamical matrix of $L 1_{2} \mathrm{Cu}_{3} \mathrm{Au}$ at the high symmetry points in the Brillouin zone. They identified the motions of atoms in various phonons, such as the highest frequency modes above 25 $\mathrm{meV}$, which involved the motions of $\mathrm{Cu}$ atoms, primarily in the plane of their cube faces, with opposing movements of $\mathrm{Au}$ atoms. The $\mathrm{Cu}-\mathrm{Cu}$ motions were found primarily in the modes around $20 \mathrm{meV}$. Nagel, et al. compared the dynamics of the ordered alloy to that of the disordered alloy calculated with the risky virtual crystal approximation, and found that the modes around $20 \mathrm{meV}$ in the ordered alloy undergo the greatest change with disorder. It was suggested that constraints on the motions of $\mathrm{Cu}$ atoms from the framework of the massive $\mathrm{Au}$ atoms, are a characteristic of the $L 1_{2}$ structure of $\mathrm{Cu}_{3} \mathrm{Au}$. These constraints were assumed to be removed in the disordered alloy, causing a shift of the modes at $20 \mathrm{meV}$ to lower energy. ${ }^{19}$ Although we do not have a clear picture of the lattice dynamics of the disordered alloys, the matched phonon DOS curves shown in Fig. 6 are consistent with this picture.

The difference of vibrational entropy between the ordered and disordered phases of $\mathrm{Cu}_{3} \mathrm{Au}$ can also be obtained by using the neutron-weight corrected entropy of the disordered alloy, rather than the disordered DOS curve of Fig. 6. The phonon entropy of disordering depends sensitively on the phonon DOS of both the ordered and disordered phases of $\mathrm{Cu}_{3} \mathrm{Au}$. The DOS of ordered $\mathrm{Cu}_{3} \mathrm{Au}$ is known more reliably than the DOS of the disordered phase, owing to the reliable neutron-weight correction of the ordered phase. ${ }^{20}$ Most of the uncertainty in the value of $\Delta S_{\mathrm{ph}}^{\mathrm{d}-\mathrm{o}}$ derived from the phonon DOS comes from the neutron-weight correction of the disordered phase. For the correction methods discussed in the previous sub-sections, the values of $\Delta S_{\mathrm{ph}}^{\mathrm{d}-\mathrm{o}}$ can be obtained from the points in Fig. 4. Using an entropy of formation for the ordered alloy of $0.05 \pm 0.03 k_{\mathrm{B}} /$ atom, ${ }^{20}$ our best estimate of the phonon entropy of disordering in $\mathrm{Cu}_{3} \mathrm{Au}$ is $0.15 \pm 0.05 k_{\mathrm{B}} /$ atom at $300 \mathrm{~K}$. This result for $S_{\mathrm{ph}}^{\mathrm{d}-\mathrm{o}}$ is consistent with the $0.14 \pm 0.05 k_{\mathrm{B}} /$ atom obtained by calorimetry, ${ }^{19}$ but somewhat larger than the $0.06 k_{\mathrm{B}}$ /atom calculated by Ozolinšs, Wolverton, and Zunger. ${ }^{8}$ The phonon entropy of disordering at $T_{\mathrm{c}}$ will be different, depending on how the phonon DOS of the two phases change between 300 and $663 \mathrm{~K}$.

\section{CONCLUSIONS}

Neutron-weighted phonon DOS curves were obtained from inelastic neutron scattering spectra measured on six disordered $\mathrm{Cu}-\mathrm{Au}$ alloys at $300 \mathrm{~K}$. The partial phonon entropies of $\mathrm{Cu}$ and $\mathrm{Au}$ are expected to vary smoothly as functions of composition. This allowed the development of neutronweight corrections to the phonon entropy using models for the partial phonon entropies of $\mathrm{Cu}$ and $\mathrm{Au}$ atoms. We tested several schemes for obtaining a phonon entropy of alloying from a neutron-weighted phonon DOS curve. These phonon entropies of formation of disordered fcc $\mathrm{Cu}-\mathrm{Au}$ alloys were generally in agreement with each other and with the results of a calculation by Ozolins $\breve{s}$, Wolverton, and Zunger using a local cluster approximation. We estimate a phonon entropy of disordering of $0.15 \pm 0.05 k_{\mathrm{B}}$ /atom for $\mathrm{Cu}_{3} \mathrm{Au}$ at $300 \mathrm{~K}$. A resonance mode associated with the motions of the heavy $\mathrm{Au}$ atoms in the $\mathrm{Cu}$-rich alloys was observed at $9 \mathrm{meV}$. This resonance mode was within a reasonable agreement with predictions of mass-defect theory.

\section{ACKNOWLEDGMENTS}

This work was supported by the U.S. Department of Energy under Contract Nos. DE-FG03-96ER45572 and BES-MS, W-31-109-ENG-38. 
${ }^{1}$ D. B. Bowen, Acta Metall. 2, 573 (1954).

${ }^{2}$ P. A. Flinn, G. M. McManus, and J. A. Rayne, J. Phys. Chem. Solids 15, 189 (1960).

${ }^{3}$ L. Anthony, J. K. Okamoto, and B. Fultz, Phys. Rev. Lett. 70, 1128 (1993).

${ }^{4}$ G. D. Garbulsky and G. Ceder, Phys. Rev. B 49, 6327 (1994).

${ }^{5}$ B. Fultz, L. Anthony, L. J. Nagel, R. M. Nicklow, and S. Spooner, Phys. Rev. B 52, 3315 (1995).

${ }^{6}$ E. Obradó, L. Mañosa, and A. Planes, Phys. Rev. B 56, 20 (1997).

${ }^{7}$ A. van de Walle, G. Ceder, and U. V. Waghmare, Phys. Rev. Lett. 80, 4911 (1998).

${ }^{8}$ V. Ozolinsš, C. Wolverton, and A. Zunger, Phys. Rev. B 58, 5897 (1998).

${ }^{9}$ P. D. Bogdanoff and B. Fultz, Philos. Mag. B 79, 753 (1999).

${ }^{10}$ P. D. Bogdanoff and B. Fultz, Philos. Mag. B 81, 299 (2001).

${ }^{11}$ V. Ozoliņš and M. Asta, Phys. Rev. Lett. 86, 448 (2001).

${ }^{12}$ C. Wolverton and V. Ozoliņš, Phys. Rev. Lett. 86, 5518 (2001).

${ }^{13}$ A. van de Walle and G. Ceder, Rev. Mod. Phys. 74, 11 (2002).

${ }^{14}$ D. T. Hawkins and R. Hultgren, J. Chem. Thermodyn. 3, 175 (1971).

${ }^{15}$ S. Katano, M. Iizumi, and Y. Noda, J. Phys. F: Met. Phys. 18, 2195 (1988).

${ }^{16}$ I. M. Lifshitz, J. Phys. USSR 7, 215 (1943); 7, 249 (1943); 8, 289 (1944).

${ }^{17}$ A. Zunger, in Statics and Dynamics of Alloy Phases, edited by P. E. A. Turchi and A. Gonis (Plenum, New York, 1994), p. 361.

${ }^{18}$ E. C. Svensson, B. N. Brockhouse, and J. M. Rowe, Phys. Rev. 155, 619 (1967).

${ }^{19}$ L. J. Nagel, L. Anthony, and B. Fultz, Philos. Mag. Lett. 72, 421 (1995).

${ }^{20}$ P. D. Bogdanoff, B. Fultz, and S. Rosenkranz, Phys. Rev. B 60, 3976 (1999).
${ }^{21}$ A. F. Yue, I. Halevy, A. Papandrew, P. D. Bogdanoff, B. Fultz, W. Sturhahn, E. E. Alp, and T. S. Toellner, Hyperfine Interact. 141, 249 (2002).

${ }^{22}$ S. C. Moss, Phys. Rev. 35, 3547 (1964).

${ }^{23}$ T. Proffen, V. Petkov, S. J. L. Billinge, and T. Vogt, Z. Kristallogr. 217, 47 (2002).

${ }^{24}$ K. F. Ludwig, Jr., G. B. Stephenson, J. L. Jordan-Sweet, J. Mainville, Y. S. Yang, and M. Sutton, Phys. Rev. Lett. 61, 1859 (1988).

${ }^{25}$ J. M. Sanchez, F. Ducastelle, and D. Gratias, Physica A 128, 334 (1984).

${ }^{26}$ B. Fultz, T. A. Stephens, E. E. Alp, M. Y. Hu, J. P. Sutter, T. S. Toellner, and W. Sturhahn, Phys. Rev. B 61, 14517 (2000).

${ }^{27} \mathrm{~V}$. Ozolins $\breve{s}$, private communication of parameters from Ref. 8

${ }^{28}$ E. C. Svensson and W. A. Kamitakahara, Can. J. Phys. 49, 2291 (1971).

${ }^{29}$ R. M. Cunningham, L. D. Muhlestein, W. M. Shaw, and C. W. Tompson, Phys. Rev. B 2, 4864 (1970).

${ }^{30}$ H. Bjerrum Møller and A. R. Mackintosh, Phys. Rev. Lett. 15, 623 (1965).

${ }^{31}$ J. W. S. Rayleigh, Theory of Sound (Dover, Mineola, NY, 1945), Sec. $88,92 a$.

${ }^{32}$ A. R. Maradudin, E. W. Montroll, G. H. Weiss, and I. P. Ipatova, Theory of Lattice Dynamics in the Harmonic Approximation, Solid State Physics Suppl. 3 (Academic Press, New York, 1971), pp. 353-514.

${ }^{33}$ R. J. Elliott and D. W. Taylor, Proc. R. Soc. London, Ser. A 296, 161 (1967).

${ }^{34}$ J. W. Lynn, H. G. Smith, and R. M. Nicklow, Phys. Rev. B 8, 3493 (1973).

${ }^{35}$ P. D. Bogdanoff, Ph.D. thesis, California Institute of Technology, 2001. 